\title{
A Novel PEGylation Method for Improving the Pharmacokinetic Properties of Anti-Interleukin-17A RNA Aptamers
}

\author{
Kazuhiko Haruta, ${ }^{1}$ Natsuki Otaki, Masakazu Nagamine,, Tomoyoshi Kayo,, Asako Sasaki, \\ Shinsuke Hiramoto,' Masayuki Takahashi, ${ }^{1}$ Kuniyoshi Hota, ${ }^{1}$ Hideaki Sato, and Hiroaki Yamazaki ${ }^{3}$
}

The obstacles to the development of therapeutic aptamers for systemic inflammatory diseases, such as nuclease degradation and renal clearance, have not been fully overcome. Here, we report a novel PEGylation method, sbC-PEGylation, which improves the pharmacokinetic properties of RNA aptamers that act against interleukin17A (IL-17A) in mice and monkeys. sbC-PEGylated aptamers were synthesized by coupling the symmetrical branching molecule 2-cyanoethyl- $N, N$-diisopropyl phosphoroamidite to the $5^{\prime}$ end of the aptamer, before conjugating two polyethylene glycol (PEG) molecules to the aptamer. Pharmacokinetic studies showed that compared with conventionally PEGylated aptamers, the sbC-PEGylated aptamer exhibited excellent stability in the blood circulation of mice and monkeys. In addition, one of the sbC-PEGylated aptamers, 17M-382, inhibited the interleukin-6 (IL-6) production induced by IL-17A in NIH3T3 cells in a concentration-dependent manner, and the half-maximal inhibitory concentration of sbC-PEGylated $17 \mathrm{M}-382$ was two times lower than that of non-PEGylated 17M-382. Furthermore, the intraperitoneal administration of sbC-PEGylated 17M-382 significantly inhibited the IL-6 production induced by IL-17A in a mouse air pouch model. Our findings suggest that the novel PEGylation method described in this study, sbC-PEGylation, could be used to develop anti-IL$17 \mathrm{~A}$ aptamers as a therapeutic option for systemic inflammatory disease.

Keywords: aptamer, interleukin-17A, PEGylation, pharmacokinetics

\section{Introduction}

A PTAMERS ARE ARTIFICIAL oligonucleotides that specifically bind to target molecules with a high affinity. Consequently, they can inhibit the binding of a protein to its receptor, similar to therapeutic neutralizing antibodies. However, aptamers are considered to have some clinical advantages over therapeutic antibodies, for example, they exhibit low immunogenicity [1-3].

A large variety of therapeutic aptamers have been reported since the systematic evolution of ligands by exponential enrichment (SELEX), a technique used to identify aptamers, was established [4,5]. However, only pegaptanib, an anti-vascular endothelial growth factor (VEGF) aptamer, has been approved for the treatment of age-related macular degeneration [6]. In addition, recent developments in this area have focused on treatments for ophthalmic disease [7]. These points indicate that the obstacles to the development of therapeutic aptamers for other diseases, especially systemic diseases, such as nuclease degradation and renal clearance, have not been fully overcome.

PEGylation, in which polyethylene glycol (PEG) is conjugated to a pharmaceutical, is one of the strategies used to inhibit the enzyme degradation and renal clearance of drugs and, hence, to improve their stability in vivo. In studies of protein-based therapeutics, it was reported that the molecular weight (MW) and configuration of PEG affected not only the pharmacokinetic (PK) properties of the drug but also its biological activity. Thus, it is important to select PEG with appropriate $\mathrm{MW}$ and configurations for the desired clinical benefits [8-11]. On the other hand, there is limited information about the PEGylation of aptamers, although various techniques involving oligonucleotide modification have been developed to protect aptamers from nuclease degradation [12-16]. In this study, to optimize the in vivo PK properties of aptamers, we developed a novel PEGylation method, symmetrical branching 2-cyanoethyl- $N, N$-diisopropyl (CED)

\footnotetext{
${ }^{1}$ R\&D Center, ${ }^{3}$ Prescription Products Development, Zenyaku Kogyo Co., Ltd., Tokyo, Japan.

${ }^{2}$ GeneDesign, Inc., Osaka, Japan.

(C) Kazuhiko Haruta, et al., 2017; Published by Mary Ann Liebert, Inc. This Open Access article is distributed under the terms of the Creative Commons License (http://creativecommons.org/licenses/by/4.0), which permits unrestricted use, distribution, and reproduction in any medium, provided the original work is properly credited.
} 
phosphoramidite PEGylation (sbC-PEGylation), and we evaluated the effects of sbC-PEGylation on the PK properties of RNA aptamers that inhibit interleukin-17A (IL-17A), which were originally reported by Ishiguro et al. [17]. It was found that sbC-PEGylation markedly improved the PK properties and enhanced the neutralizing activity of anti-IL-17A aptamers. These results suggest that sbC-PEGylation might represent a breakthrough in the development of therapeutic aptamers.

\section{Materials and Methods}

\section{PEGylation and oligonucleotides}

The PEGylation and synthesis of the oligonucleotides were performed by GeneDesign, Inc. (Osaka, Japan). The PEG molecules used in this study were obtained from NOF Corporation (Tokyo, Japan) or JenKem Technology (Beijing, China). Symmetrical branching CED phosphoramidite, DMTtriethyloxy-glycol phosphoramidite (ChemGenes Corporation, Wilmington, MT), and ssH linker phosphoramidite (Sigma-Aldrich Corporation, St. Louis, MO) were used for the sbC-PEGylation of the anti-IL-17A aptamers, according to the scheme described in Fig. 1. In brief, the $5^{\prime}-\mathrm{OH}$ oligonucleotide (compound 2) was sequentially synthesized from a 3 '-inverted deoxythymidine (idT)-loaded controlled pore glass that served as a starting material by using an automated solid-phase oligonucleotide synthesizer, before being subjected to acid detritylation. In the same synthesizer, symmetrical branching CED phosphoramidite (compound 1, 6 equivalents) was reacted with compound 2 to give compound $\mathbf{3}$, which had a $5^{\prime}-\mathrm{O}-$ branched oligonucleotide. After oxidation and deprotection of compound $\mathbf{3}$, the resultant compound $\mathbf{4}$ was reacted with DMT-triethyloxy-glycol phosphoramidite (compound 5, 9 equivalents), giving compound $\mathbf{6}$. Oxidation and deprotection of compound $\mathbf{6}$ proceeded in the same manner as described earlier, and the resultant compound 7 was reacted with $\mathrm{ssH}$ linker phosphoramidite (compound $\mathbf{8}, 9$ equivalents) and oxidized successively, giving compound $\mathbf{9}$. Then, compound 9 was cleaved from the resin and base deprotected by using ammonium hydroxide. Next the 2'-tert-butyldimethylsilyl (TBDMS) group was deprotected with acid, and then the product was purified by using reverse-phase column chromatography to give the purified amine compound 10, which was used as a PEGylation precursor. Next, an aqueous solution of compound $\mathbf{1 0}$ and a dimethyl sulfoxide-acetonitrile (4:1) solution of activated PEG (compound 11, 9 equivalent) were added to $0.1 \mathrm{M}$ sodium carbonate buffer solution ( $\mathrm{pH} 9.0$ ), and the mixture was stirred at $25^{\circ} \mathrm{C}$ for $2 \mathrm{~h}$ to give the crude PEGylated product. After the crude PEGylated product had been purified with reverse-phase column chromatography, the desired purified PEGylated aptamers were obtained. In some studies, compound $\mathbf{2}$ was conventionally PEGylated by using ssH linker phosphoramidite alone. The weights of the sbCPEGylated and PEGylated aptamers were calculated as oligonucleotide weight values.

The RNA sequence of the anti-IL-17A aptamers (17M200-S1 and 17M-382) used in this study, 5'-GGGUA GCCGGAGGAGTCAGTAAUCGGUACCC-3', was based on the Apt21-2 sequence reported by Ishiguro et al. [17]. To
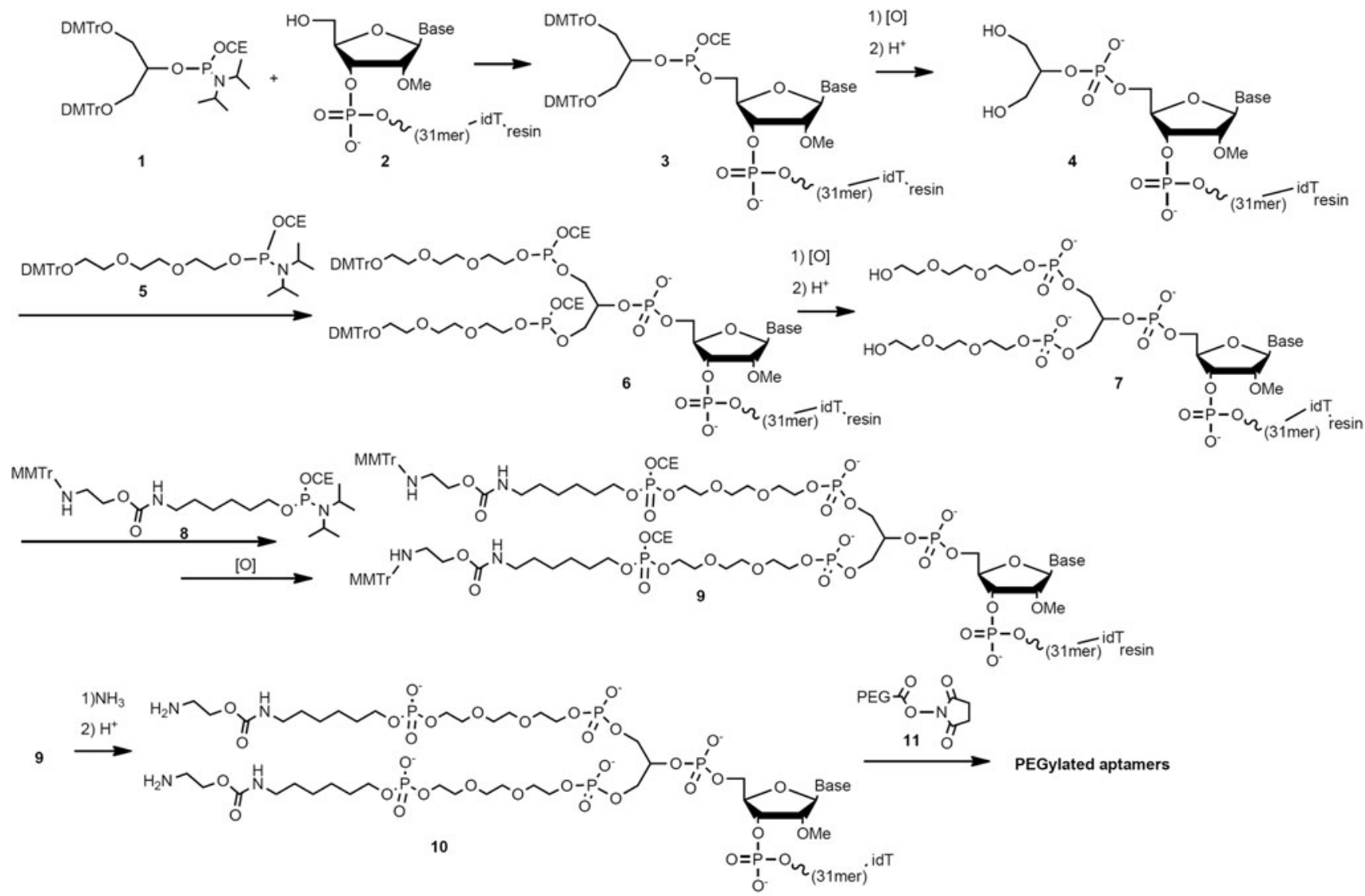

FIG. 1. Scheme for sbC-PEGylation. DMTr, 4,4'-dimethoxytrityl; MMTr, p-methoxyphenyl diphenylmethyl; OCE, cyanoethoxy. 
prevent nuclease degradation, fluorine, methoxy, or hydrogen groups were introduced at the $2^{\prime}-\mathrm{OH}$ positions of some riboses, and the aptamers' phosphodiester backbones were partially replaced with phosphorothioate.

\section{PK studies in mice}

The PK studies were performed according to the procedures approved by the Zenyaku Kogyo Co., Ltd., animal care and use committee. Male C57BL/6J mice (6 weeks old) were purchased from Charles River Laboratories, Japan (Kanagawa, Japan). They were maintained at around $22^{\circ} \mathrm{C}$ under a $12 \mathrm{~h}$ light/dark cycle and given standard chow and tap water ad libitum. sbC-PEGylated or PEGylated aptamers were dissolved in saline at a concentration of $0.2 \mathrm{mg} / \mathrm{mL}$. They were intravenously administered to the mice at a dose of $1 \mathrm{mg} / \mathrm{kg}$. Blood was collected from the mice at the indicated time points ( $n=3$ per time point), and ethylenediaminetetraacetic acid (EDTA) plasma samples were prepared and stored at $-70^{\circ} \mathrm{C}$ until the assays.

\section{PK studies in monkeys}

The PK studies in cynomolgus monkeys were conducted at Ina Research, Inc. (Nagano, Japan), according to the procedures approved by the Ina Research, Inc., institutional animal care and use committee. Male cynomolgus monkeys (age: 35 years; body weight: $2.6-4.4 \mathrm{~kg}$ ) were used in this study. sbC-PEGylated or conventionally PEGylated 17M-382 was dissolved in saline at a concentration of $1 \mathrm{mg} / \mathrm{mL}$. The aptamers were intravenously administered to the monkeys $(n=3)$ at a dose of $1 \mathrm{mg} / \mathrm{kg}$. Two weeks after their intravenous administration, they were also subcutaneously administered at a dose of $1 \mathrm{mg} / \mathrm{kg}$. Blood was collected at the indicated time points, and EDTA plasma samples were prepared and stored at $-70^{\circ} \mathrm{C}$ until the assays.

\section{Enzyme-linked oligo-sorbent assay}

The plasma levels of aptamers were measured by using an enzyme-linked oligo-sorbent assay (ELOSA) according to the method reported by Healy et al. [18]. The RNA sequences of the detection probe and capture probe were 5'-6-FAMspacer 18-GGGUACCGAUUACUG-3' and 5'-ACUCCUC CGGCUACCC-spacer 9-amino C6 linker-3', respectively. The PK parameters were derived by using noncompartmental models with WinNonlin ${ }^{\circledR}$ version 6.4 on Phoenix ${ }^{\circledR} 1.4$ (Pharsight Corp., Mountain View, CA).

\section{In vitro IL-17A-neutralizing activity}

The effects of sbC-PEGylation on the IL- 6 production induced by human IL-17A in NIH3T3 cells were examined. Namely, $10 \mathrm{ng} / \mathrm{mL}$ of human IL-17A (R\&D Systems, Inc., Minneapolis, MN) was incubated with various concentrations of the aptamers for $30 \mathrm{~min}$ at $37^{\circ} \mathrm{C}$. Then, suspensions of NIH3T3 cells (American Type Culture Collection, Manassas, VA) (density: $5 \times 10^{5}$ cells $/ \mathrm{mL}$ ) and mouse tumor necrosis factor (TNF)- $\alpha(2 \mathrm{ng} / \mathrm{mL}$; R\&D Systems, Inc.) were added to the plates. After $24 \mathrm{~h}$, the supernatants were collected and stored at $-70^{\circ} \mathrm{C}$ until the assays were performed. Then, the levels of mouse IL-6 in the supernatants were measured by using an ELISA kit (Invitrogen Corporation, Frederick, MD).

\section{In vivo IL-17A-neutralizing activity}

According to the method reported by Maione et al. [19], we examined the effects of sbC-PEGylation on the IL-17Aneutralizing activity of $17 \mathrm{M}-382$ by using a mouse air pouch model. sbC-PEGylated 17M-382 was intraperitoneally administered at 1,24,72, or $168 \mathrm{~h}$ before the administration of human IL-17A ( $500 \mu \mathrm{g} /$ pouch, kindly donated by Dr. Ishiguro, Tokyo University) into each air pouch. Then, the exudates from each air pouch were collected at $24 \mathrm{~h}$ after administration of human IL-17A and they were stored at $-70^{\circ} \mathrm{C}$ until the concentration of IL- 6 was measured by using an ELISA kit (Invitrogen Corporation).

\section{Statistical analysis}

Data are expressed as the mean \pm standard deviation (SD, $n=3$, for the PK studies and in vitro studies) or the mean \pm standard error of the mean (SEM, $n=7$ or 8 , for the mouse air pouch model). The statistical significance of differences was assessed by using one-way analysis of variance (ANOVA) followed by Dunnett's test. These statistical analyses and the determination of half-maximal inhibitory concentration $\left(\mathrm{IC}_{50}\right)$ values (using the four-parameter curve fit algorithm) were performed by using the Kaleida Graph 3.6 software (Synergy Software, Reading, PA). $P$-values of $<0.05$ were considered significant.

\section{Results \\ sbC-PEGylation}

Figure 2A shows the results of the reverse-phase high performance liquid chromatography (HPLC) analysis of 17M382 after it had been sbC-PEGylated with two-armed $40-\mathrm{kDa}$ PEG (Y-shaped PEG; JenKem Technology). The yields of sbC-PEGylated 17M-200-S1 and sbC-PEGylated 17M-382 were $85.0 \%$ and $87.4 \%$, respectively. After purifying them with reverse-phase HPLC, we confirmed that the sbCPEGylated aptamers contained two 40-kDa PEG molecules using matrix-assisted laser desorption/ionization time-offlight mass spectrometry (MALDI-TOF-MS, Fig. 2B).

\section{Effects of sbC-PEGylation on the PK properties of aptamers in mice}

To evaluate the effects of sbC-PEGylation on the renal clearance and nuclease degradation of aptamers in vivo, we first used 17M-200-S1, which was easily degraded in mouse serum (Supplementary Fig. S1; Supplementary Data are available online at www.liebertpub.com/nat). sbCPEGylated 17M-200-S1 had the longest plasma half-life $\left(\mathrm{t}_{1 / 2}\right.$, $22.5 \mathrm{~h}$ ) of the aptamers that were conjugated with $80-\mathrm{kDa}$ PEG. The plasma $t_{1 / 2}$ of sbC-PEGylated 17M-200-S1 was approximately two to three times longer than those of the two-armed 80-kDa PEG (7.5 h) and the four-armed $80-\mathrm{kDa}$ PEG (10.5 h). Compared with the aptamers that were PEGylated with 40-kDa PEG, the sbC-PEGylated 17M-200-S1 remained in circulation for 4.5 to 6.0 times longer (Fig. 3 and Table 1).

Next, we used 17M-382, which was not degraded in mouse serum for $72 \mathrm{~h}$ (Supplementary Fig. S1), to evaluate the effects of sbC-PEGylation on renal clearance. The plasma $t_{1 / 2}$ of sbC-PEGylated 17M-382 (22.8 h) and sbC-PEGylated 

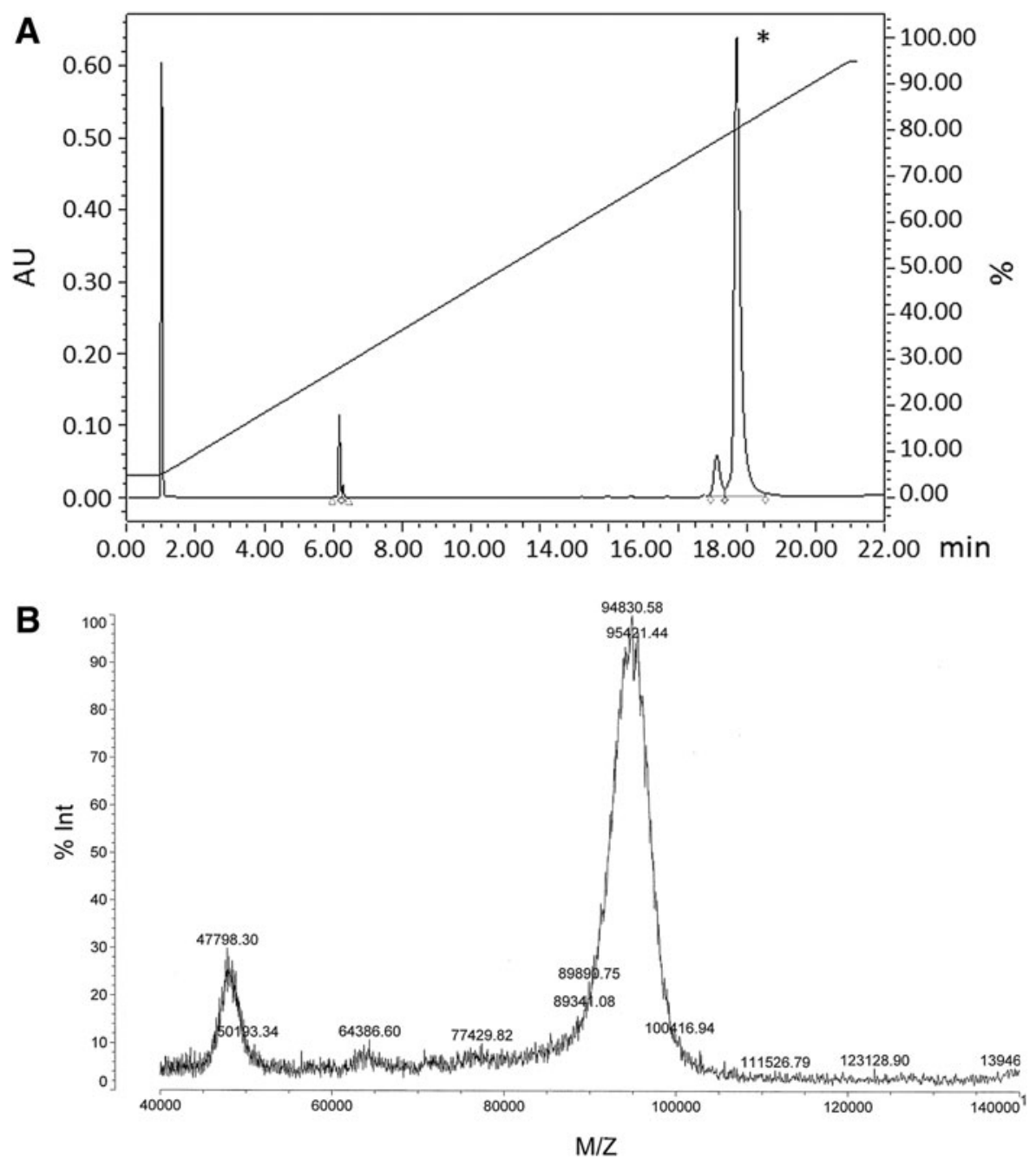

FIG. 2. (A) Reverse-phase HPLC and (B) matrix-assisted laser desorption/ionization time-of-flight mass spectrometry (MALDI-TOF-MS) analyses of sbC-PEGylated 17M-382. *The yield of sbC-PEGylated $17 \mathrm{M}$ 382 was $87.4 \%$.

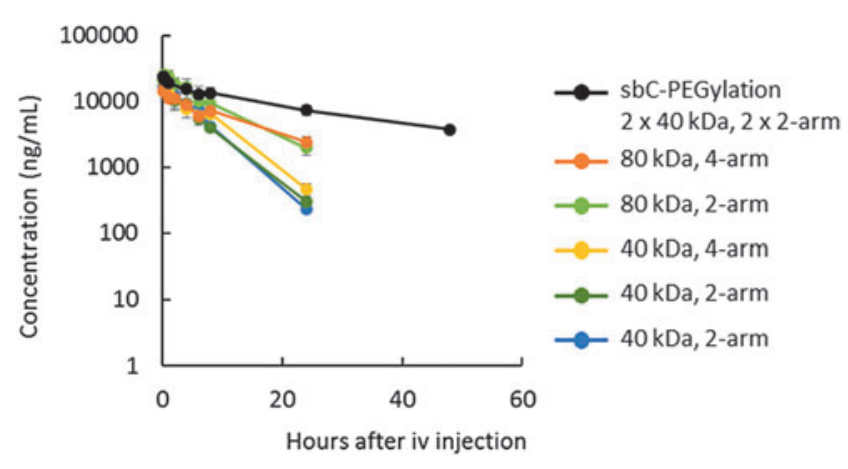

FIG. 3. Plasma concentration-time curves of sbC-PEGylated and conventionally PEGylated aptamers after their intravenous injection into mice. 17M-200-S1 that had been sbCPEGylated with two 2-armed 40-kDa PEG (Y-shaped PEG, block) or conventionally PEGylated with various PEG (orange: SUNBRIGHT $^{\circledR}$ GL4-800GS2; yellow-green: SUNBRIGHT GL2-800GS2; yellow: SUNBRIGHT GL4-400GS2; green: SUNBRIGHT GL2-400GS2; blue: Y-shaped PEG) was intravenously administered to mice at a dose of $1 \mathrm{mg} / \mathrm{kg}$. Three mice were used at each time point, and the data are expressed as mean \pm SD values $(n=3)$.
17M-200-S1 (22.5 h) did not differ virtually. In addition, the plasma $\mathrm{t}_{1 / 2}$ of sbC-PEGylated $17 \mathrm{M}-382$ was almost as long as that of 17M-382 PEGylated with four-armed 80-kDa PEG (20.9 h, Table 1). However, the use of linear 40-kDa PEG instead of two-armed PEG markedly reduced the plasma $t_{1 / 2}$ of sbC-PEGylated 17M-382 (4.9 h). Taken together, it was considered that sbC-PEGylation with two 2-armed 40-kDa PEG molecules increased the aptamers' MW to a greater extent (total: $80 \mathrm{kDa}$ ) and induced more marked steric hindrance (caused by the 4 arms around the aptamers) than conventional PEGylation, resulting in reductions in renal clearance and nuclease degradation.

\section{Effects of sbC-PEGylation on the PK properties of aptamers in monkeys}

Since it was predicted that the most suitable oligonucleotide/PEGylation combination would optimize the aptamers' PK properties in monkeys, we used 17M-382, and we compared the PK properties of sbC-PEGylated aptamers with those of conventionally PEGylated aptamers. When sbCPEGylated 17M-382 was administered intravenously and subcutaneously, it exhibited plasma $t_{1 / 2}$ of $72.0 \pm 10.3$ and $107 \pm 52.8 \mathrm{~h}$, respectively, which were 4.1 and 6.5 times longer than those obtained for 17M-382 PEGylated with 
Table 1. Plasma Half-Lives $\left(\mathrm{T}_{1 / 2}\right)$ of SBC-Pegylated and Pegylated Aptamers in Mice

\begin{tabular}{|c|c|c|c|c|}
\hline \multirow[b]{2}{*}{ Aptamers } & \multicolumn{3}{|l|}{$P E G$} & \multirow[b]{2}{*}{$t_{1 / 2}(h)$} \\
\hline & Product name & $M W(k D a)$ & No. of arms & \\
\hline sbC-PEGylated 17M-200-S1 & Y-shaped PEG+Y-shaped PEG & $80(40+40)$ & $4(2+2)$ & 22.5 \\
\hline \multirow[t]{5}{*}{ PEGylated 17M-200-S1 } & SUNBRIGHT $^{\circledR}$ GL4-800GS2 & 80 & 4 & 10.5 \\
\hline & SUNBRIGHT GL2-800GS2 & 80 & 2 & 7.5 \\
\hline & SUNBRIGHT GL4-400GS2 & 40 & 4 & 5.0 \\
\hline & SUNBRIGHT GL2-400GS2 & 40 & 2 & 4.3 \\
\hline & Y-shaped PEG & 40 & 2 & 3.7 \\
\hline \multirow[t]{2}{*}{ sbC-PEGylated 17M-382 } & Y-shaped PEG+Y-shaped PEG & $80(40+40)$ & $4(2+2)$ & 22.8 \\
\hline & $\begin{array}{l}\text { SUNBRIGHT ME-400GS+SUNBRIGHT } \\
\text { ME-400GS }\end{array}$ & $80(40+40)$ & 2 (linear+linear) & 4.9 \\
\hline PEGylated 17M-382 & SUNBRIGHT GL4-800GS2 & 80 & 4 & 20.9 \\
\hline
\end{tabular}

SUNBRIGHT $^{\circledR}$ is a registered trademark of the NOF Corporation. MW, molecular weight.

two-armed $40 \mathrm{kDa}$ PEG, respectively. Similarly, the area under the curve values of sbC-PEGylated 17M-382 were 5.3 to 5.5 times longer than those of PEGylated 17M-382. On the contrary, the CL/F value of sbC-PEGylated $17 \mathrm{M}-382$ was approximately one-fifth lower than that of PEGylated 17M382. When the aptamers were administered subcutaneously, the $\mathrm{T}_{\max }$ of sbC-PEGylated 17M-382 was 3.8 times longer than that of PEGylated 17M-382. However, there were no differences in $\mathrm{C}_{\max }, \mathrm{Vz} / \mathrm{F}$, or Vss (intravenous administration) between the aptamers (Fig. 4 and Table 2).

\section{The effects of sbC-PEGylation on the in vitro neutralizing activity of $17 M-382$}

Since the PEGylation of pharmaceuticals sometimes reduces their biological activity [10], it is possible that sbCPEGylation might decrease the neutralizing activity of the anti-IL-17A aptamers. Thus, we examined the effects of sbCPEGylation on the neutralizing activity of $17 \mathrm{M}-382$. NonPEGylated 17M-382 inhibited the IL-6 production induced by IL-17A in a concentration-dependent manner (Fig. 5), and its $\mathrm{IC}_{50}$ value was $19.7 \pm 6.3 \mathrm{ng} / \mathrm{mL}$. On the other hand, the $\mathrm{IC}_{50}$ value of sbC-PEGylated $17 \mathrm{M}-382$ was $10.0 \pm 1.0 \mathrm{ng} /$ $\mathrm{mL}$. These results indicate that rather than reducing the in vitro neutralizing activity of the aptamers, sbC-PEGylation enhanced it by about twofold.

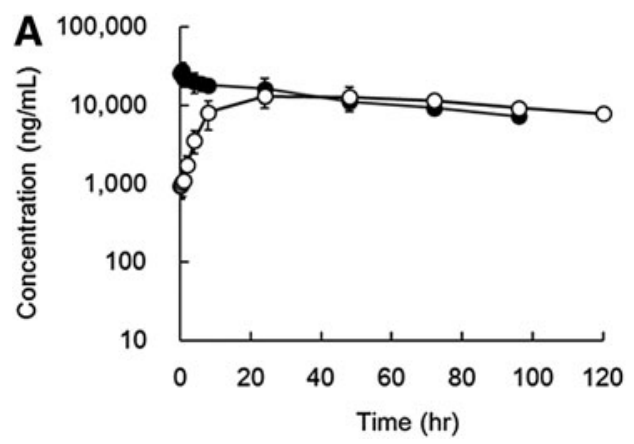

The effects of sbC-PEGylation on the in vivo neutralizing activity of the aptamers

Next, we examined the effects of sbC-PEGylation on in vivo IL-17A-neutralizing activity by using a mouse air pouch model. In the saline-treated group, the IL- 6 concentration of the air pouch was increased at $24 \mathrm{~h}$ after the administration of human IL-17A. However, the intraperitoneal administration of sbC-PEGylated 17M-382 $1 \mathrm{~h}$ before the administration of IL-17A reduced the IL- 6 concentration of the air pouch in a dose-dependent manner (Fig. 6A). Significant differences were observed between the IL-17A-neutralizing activity induced by 1 and $3 \mathrm{mg} / \mathrm{kg}$ of sbC-PEGylated 17M-382. Moreover, sbC-PEGylated 17M-382 continued to exhibit in vivo neutralizing activity for $72 \mathrm{~h}$; that is, $3 \mathrm{mg} / \mathrm{kg}$ of sbCPEGylated 17M-382 significantly reduced the IL-6 concentration of the air pouch (Fig. 6B). These findings indicate that sbC-PEGylated aptamers diffuse into inflamed tissues and that the in vivo neutralizing activity of sbC-PEGylated aptamers can be maintained for several days.

\section{Discussion}

In this study, we reported a novel PEGylation method, sbC-PEGylation, which markedly improved the PK parameters and in vitro neutralizing activity of anti-IL-17A aptamers. Moreover, it was indicated that sbC-PEGylated

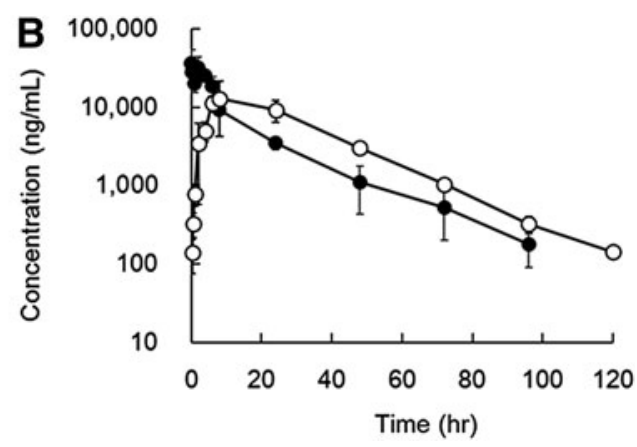

FIG. 4. Plasma concentration-time curves of sbC-PEGylated and conventionally PEGylated aptamers after their injection into monkeys. 17M-382 that had been sbC-PEGylated with two 2-armed 40-kDa PEG (A) or conventionally PEGylated with a two-armed 40-kDa PEG (B, SUNBRIGHT GL2-400GS2) was intravenously (closed circles) or subcutaneously (open circles) administered to monkeys at a dose of $1 \mathrm{mg} / \mathrm{kg}$. Data are expressed as mean \pm SD values $(n=3)$. 


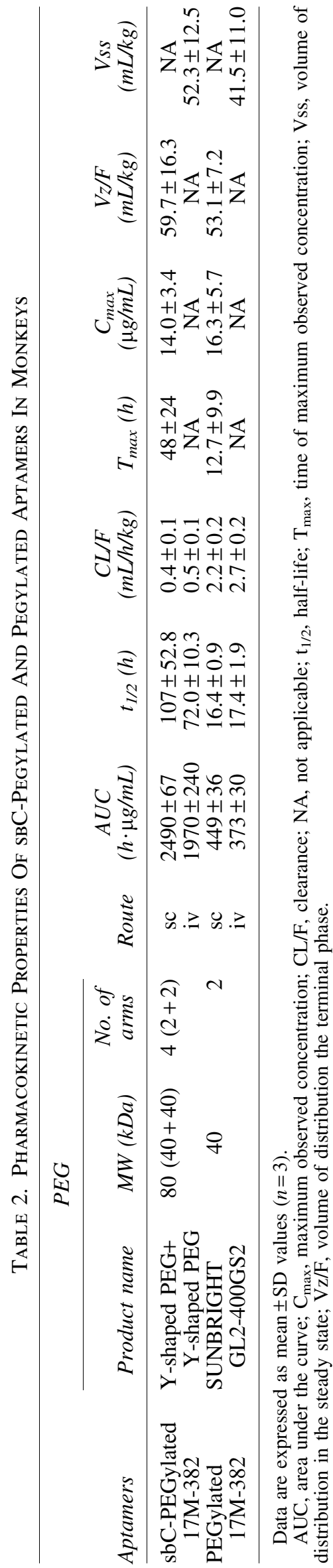

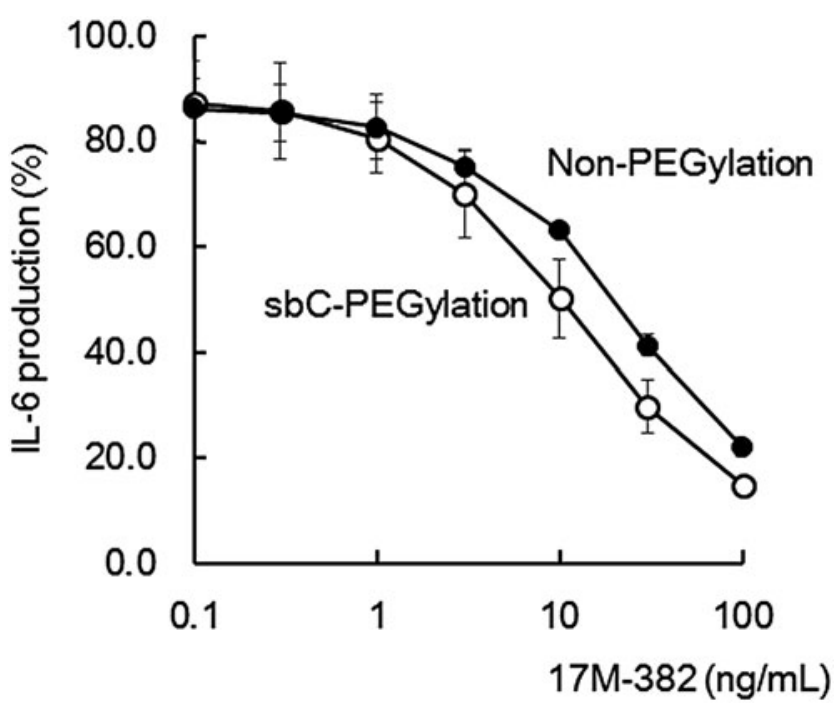

FIG. 5. In vitro neutralizing activity of sbC-PEGylated aptamers. The IL-6 production induced by human IL-17A in NIH3T3 cells was inhibited by sbC-PEGylated 17M-382 (open circles) and non-PEGylated 17M-382 (closed circles). Data are expressed as the mean $\pm S D$ values of three independent experiments.

aptamers diffuse into inflamed tissue and that they continue to exhibit in vivo neutralizing activity for $72 \mathrm{~h}$ after their administration. To the best of our knowledge, this is the first report to indicate that performing PEGylation with both symmetrical branching CED phosphoramidite and twoarmed 40-kDa PEG improves the PK properties of aptamers, although Kang et al. reported that the conjugation of two linear PEG molecules and a symmetrical doubler prolonged the in vitro whole blood clotting time of an anti-thrombin DNA aptamer [20].

Figure 3 and Table 1 suggest that the MW of PEG (total: $80 \mathrm{kDa}$ ) and the steric hindrance induced by two 2 -armed PEG inhibit the renal clearance and nuclease degradation of aptamers. Since the $t_{1 / 2}$ of $170-k D a$ PEG in circulation was only 1.4 times longer than that of 50-kDa PEG [21], it was suggested that the conjugation of $80-\mathrm{kDa}$ PEG might be an inefficient way of prolonging the plasma $t_{1 / 2}$ of aptamers. However, the half-life of 17M-200-S1 that had been PEGylated with $80-\mathrm{kDa}$ PEG was two times longer than that of 17M-200-S1 that had been PEGylated with 40-kDa PEG (Table 1). These results suggested that the use of two 40-kDa PEG might be responsible for improvement of PK properties by sbC-PEGylation. As for the number of arms, compared with two-armed PEG, there were no advantages of using four-armed PEG molecules when PEG was conjugated to aptamers by using conventional methods. On the other hand, the half-life of 17M-200-S1 that had been sbC-PEGylated with two 2-armed 40-kDa PEG molecules (total: four-armed $80-\mathrm{kDa}$ PEG) was two times longer than that of $17 \mathrm{M}-200-\mathrm{S} 1$ that had been PEGylated with four-armed 80-kDa PEG. These differences between sbC-PEGylation and PEGylation were not obvious when $17 \mathrm{M}-382$, a stable oligonucleotide, was used. In addition, the plasma $t_{1 / 2}$ of $17 \mathrm{M}-382$ was markedly reduced when it was sbC-PEGylated with two linear 40-kDa PEG molecules. These results indicate that the 4 arms formed by sbC-PEGylation induce greater steric 

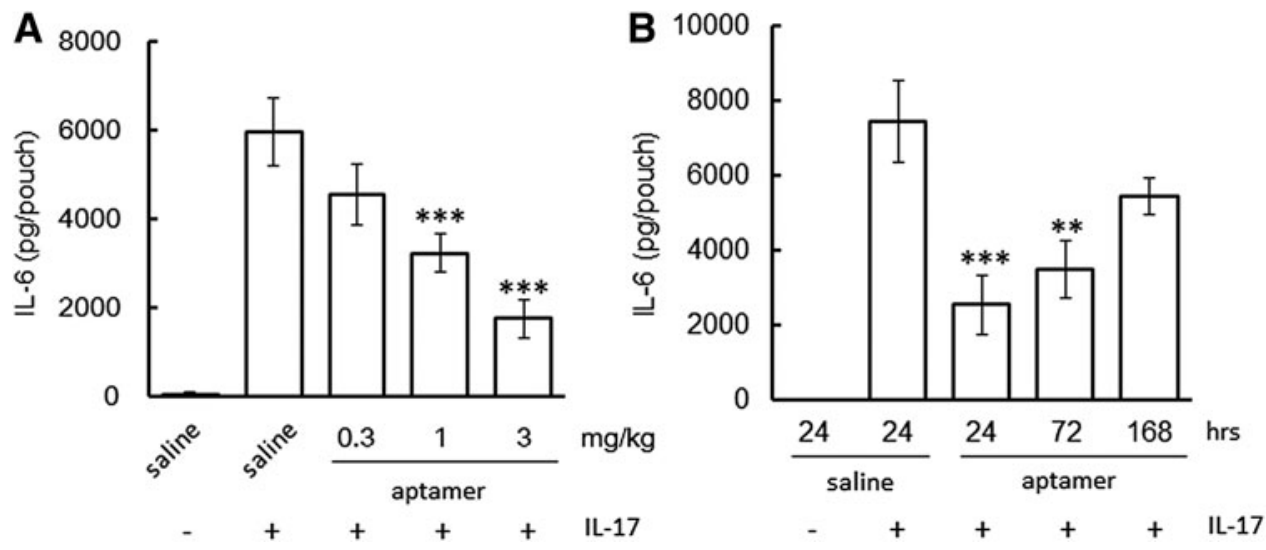

FIG. 6. In vivo neutralizing activity of anti-IL-17A aptamers. The IL-6 production induced in air pouches by human IL17A was inhibited by the intraperitoneal administration of sbC-PEGylated 17M-382. The aptamers were administered to the mice at $1 \mathrm{~h} \mathrm{(A)}$ or 24,72 , or $168 \mathrm{~h} \mathrm{(B)} \mathrm{before} \mathrm{the} \mathrm{administration} \mathrm{of} \mathrm{human} \mathrm{IL-17A} \mathrm{into} \mathrm{the} \mathrm{air} \mathrm{pouches.} \mathrm{Data} \mathrm{are} \mathrm{expressed} \mathrm{as}$ mean \pm SEM values $(n=7$ or 8$)$. $* * P<0.01$, $* * * P<0.001$ compared with the saline+IL-17A-treated group (ANOVA followed by Dunnett's test). ANOVA, analysis of variance.

hindrance around oligonucleotides and prevent aptamers from being subjected to nuclease degradation.

PEGylation sometimes inhibits the biological activity of pharmaceuticals, although the loss of biological activity by PEGylation can be ameliorated by the associated increase in the molecule's half-life. It was reported that the PEGylation of an anti-VEGF aptamer reduced its in vitro biological activity by $25 \%$ [10]. On the other hand, the steric hindrance induced by sbC-PEGylation might have enhanced the binding of the anti-IL-17A aptamer to IL-17A, and hence, increased its in vitro neutralizing activity (Fig. 5). Ishiguro et al. also found that the PEGylation of Apt21-2 with a twoarmed 40-kDa PEG molecule, SUNBRIGHT ${ }^{\circledR}$ GL2-400GS2, increased its in vitro neutralizing activity against mouse IL17A [17]. In addition, the in vitro neutralizing activity of antiIL-17A aptamers against human IL-17A improved after their PEGylation with various kinds of PEG, including SUNBRIGHT GL2-400GS2, GL2-800GS2, and GL4-800GS2 (data not shown). Therefore, it was suggested that PEG might directly interact with IL-17A. Importantly, sbC-PEGylation was able to increase the in vivo pharmacological activity of anti-IL-17A aptamers by enhancing their neutralizing activity and prolonging their half-lives.

Figure 6A indicates that the sbC-PEGylated aptamers were able to easily diffuse into inflamed tissues after their administration. Aptamers are so small that they are able to recognize epitopes that antibodies cannot access [22]. In addition, it was reported that aptamers are able to efficiently penetrate into biological compartments [2]. Namely, their small size is one of the advantages that aptamers have over therapeutic antibodies. Based on PK studies, we used 80-kDa PEG (total MW of aptamer: $92 \mathrm{kDa}$ ) to prolong the half-life of aptamers in vivo. As a result, we found that the steric hindrance induced by sbC-PEGylation with two 2-armed 40$\mathrm{kDa}$ PEG seemed to be greater than that associated with the use of $80-\mathrm{kDa}$ PEG. In fact, the $\mathrm{T}_{\max }(48 \mathrm{~h})$ of sbC-PEGylated $17 \mathrm{M}-382$ was 3.8 times longer than that $(12.7 \mathrm{~h})$ of PEGylated $17 \mathrm{M}-382$ when they were administered to monkeys subcutaneously. However, since there were no differences in $\mathrm{C}_{\max }, \mathrm{Vz} / \mathrm{F}$, or Vss between the sbC-PEGylated and PEGylated aptamers, it was suggested that sbC-PEGylated apta- mers diffuse through the body in the same way as PEGylated aptamers. In particular, the sbC-PEGylated 17M-382 inhibited the production of IL- 6 in air pouches when it was administered intraperitoneally $1 \mathrm{~h}$ before the injection of IL17A (Fig. 6A). As for therapeutic antibodies, certolizumab pegol, a PEGylated Fab' fragment of an anti-TNF- $\alpha$ antibody (total MW: $90 \mathrm{kDa}$ ), is able to penetrate inflammatory tissue more easily than other whole anti-TNF- $\alpha$ antibodies (total MW: $150 \mathrm{kDa}$, ref. 23). Taken together, it was suggested that the sbC-PEGylation of aptamers with $80-\mathrm{kDa}$ PEG does not affect their ability to penetrate into inflammatory tissues.

sbC-PEGylation might be one way of overcoming the limitations associated with the modification of ribose and/or phosphate backbones after conventional SELEX, which are used to enhance nuclease resistance. Since these modifications sometimes reduce the pharmacological activity of aptamers, not all of the nucleotides of an aptamer can be chemically modified. In the case of anti-IL-17A aptamers, we synthesized $>400$ chemically modified aptamers and examined their in vitro neutralizing activity and stability in mouse serum. As a result, we identified 17M-382, in which 2 of its 31 nucleotides cannot be chemically modified. Disappointingly, the half-life of $17 \mathrm{M}-382$ that had been conjugated to a conventional two-armed 40-kDa PEG molecule was only $17 \mathrm{~h}$ when it was administered to monkeys subcutaneously or intravenously. When it was compared with ARC15105, an anti-von Willebrand factor aptamer that has been PEGylated with 40-kDa PEG, which was selected by variant $\operatorname{SELEX~}\left(\mathrm{t}_{1 / 2}\right.$ : $67 \mathrm{~h}$, iv; $65 \mathrm{~h}$, sc; refs. 24 and 25), it was found that chemical modification could not completely prevent $17 \mathrm{M}-382$ from undergoing nuclease degradation in monkeys. However, sbC-PEGylation markedly prolonged the half-life of $17 \mathrm{M}$ $382\left(\mathrm{t}_{1 / 2}: 72 \mathrm{~h}, \mathrm{iv} ; 107 \mathrm{~h}, \mathrm{sc}\right)$, which was consistent with the findings obtained for ARC15105.

IL-17A is a cytokine that is mainly produced by $\mathrm{T}$ helper 17 cells and plays a pathogenic role in systemic inflammatory diseases, such as multiple sclerosis, rheumatoid arthritis, psoriasis, etc. [26]. Thus, it is clear that anti-IL-17A aptamers have therapeutic potential as treatments for systemic inflammatory diseases. In fact, anti-IL-17A and anti-IL-17 receptor A aptamers inhibited the progression of experimental 
autoimmune encephalomyelitis (EAE), glucose-6-phosphate isomerase (GPI)-induced arthritis, collagen-induced arthritis, and osteoarthritis in mice $[17,27,28]$. It was also reported that the administration of anti-IL-17A antibodies ameliorated the symptoms of EAE, GPI-induced arthritis, and IL-23-induced dermatitis in mice [29-31], and clinical trials of anti-IL-17A antibodies, such as secukinumab and ixekizumab, revealed that the neutralization of IL-17A was successful as a therapy for psoriasis and ankylosing spondylitis (AS) in humans [3234]. Since aptamers are considered to have some clinical advantages over therapeutic antibodies [1-3], anti-IL-17A aptamers might be an alternative to anti-IL-17A therapeutic antibodies. In particular, after its subcutaneous administration to monkeys, sbC-PEGylated 17M-382 exhibited a plasma $t_{1 / 2}$ of $107 \mathrm{~h}$, which was longer than the plasma $t_{1 / 2}$ of albinterferon (human serum albumin-interferon $\alpha$ ) in monkeys (91-92 h, ref. 35) and etanercept (a TNF receptor-Ig fusion protein) in humans $(80 \mathrm{~h}$, ref. 36$)$. It is strongly suggested that sbC-PEGylation is a useful method for developing therapeutic anti-IL-17A aptamers for systemic inflammatory diseases, such as psoriasis and AS.

In conclusion, we report that sbC-PEGylation, a novel PEGylation method, improved the PK properties of anti-IL17A aptamers. This study also suggests that the creation of new configurations using branching molecules (or doublers), spacers, and PEG could improve the PK properties of aptamers. In addition to modern oligonucleotide modification techniques, the use of novel PEGylation methods, such as sbC-PEGylation, could aid the development of therapeutic aptamers, especially for systemic disease.

\section{Acknowledgments}

The authors would like to thank Drs. Yoshikazu Nakamura, Shin Miyakawa, Masatoshi Fujiwara (all Ribomic, Inc.), and Akira Ishiguro (Tokyo University) for their advice on the oligonucleotide modification of anti-IL-17A aptamers and the establishment of the ELOSA. In addition, they gratefully acknowledge Shigeyuki Mori, Tomoyo Kimijima and Junna Takagawa (Zenyaku Kogyo Co., Ltd.) for their kind help and administrative support during this study.

\section{Author Disclosure Statement}

K.H., N.O., M.N., T.K., A.S., S.H., M.T., K.H., and H.Y. are employees of Zenyaku Kogyo Co., Ltd. H.S. is an employee of GeneDesign, Inc.

\section{References}

1. Bruno JG. (2015). Predicting the uncertain future of aptamer-based diagnostics and therapeutics. Molecules 20: 6866-6887.

2. Sun $\mathrm{H}$ and Y Zu. (2015). A highlight of recent advances in aptamer technology and its application. Molecules 20:1195911980.

3. Keefe AD, S Pai and A Ellington. (2010). Aptamers as therapeutics. Nat Rev Drug Discov 9:537-550.

4. Tuerk C and L Gold. (1990). Systematic evolution of ligands by exponential enrichment: RNA ligands to bacteriophage T4 DNA polymerase. Science 249:505-510.

5. Ellington AD and JW Szostak. (1990). In vitro selection of RNA molecules that bind specific ligands. Nature 346:818-822.
6. Ng EWM, DT Shima, P Calias, ET Cunningham Jr, DR Guyer and AP Adamis. (2006). Pegaptanib, a targeted antiVEGF aptamer for ocular vascular disease. Nat Rev Drug Discov 5:123-132.

7. Burke PA. (2015). The ups and downs of clinical translation of new technologies: Déjà vu all over again. Mol Ther 23:791-792.

8. Roberts MJ, MD Bentley and JM Harris. (2002). Chemistry for peptide and protein PEGylation. Adv Drug Delivery Rev 54:459-476.

9. Harris JM and RB Chess. (2003). Effect of pegylation on pharmaceuticals. Nat Rev Drug Discov 2:214-221.

10. Fishburn CS. (2008). The pharmacology of PEGylation: balancing PD with PK to generate novel therapeutics. J Pharm Sci 97:4167-4183.

11. Li XQ, JD Lei, ZG Su and GH Ma. (2007). Comparison of bioactivities of monopegylated rhG-CSF with branched and linear mPEG. Process Biochem 42:1625-1631.

12. Schmidt KS, S Borkowski, J Kurreck, AW Stephens, R Bald, M Hecht, M Friebe, L Dinkelborg and VA Erdmann. (2004). Application of locked nucleic acids to improve aptamer in vivo stability and targeting function. Nucleic Acids Res 32:5757-5765.

13. Ozer A, Pagano JM and Lis JT. (2014). New technologies provide quantum changes in the scale, speed, and success of SELEX methods and aptamer characterization. Mol Ther Nucleic Acids 3:e183.

14. Burmeister PE, SD Lewis, RF Silva, JR Preiss, LR Horwitz, PS Pendergrast, TG McCauley, JC Kurz, DM Epstein, C Wilson and AD Keefe. (2005). Direct in vitro selection of a 2'-O-methyl aptamer to VEGF. Chem Biol 12:25-33.

15. Gupta S, M Hirota, SM Waugh, I Murakami, T Suzuki, M Muraguchi, M Shibamori, Y Ishikawa, TC Jarvis, et al. (2014). Chemically modified DNA aptamers bind interleukin6 with high affinity and inhibit signaling by blocking its interaction with interleukin-6 receptor. J Biol Chem 289: 8706-8719.

16. Vater A and S Klussmann. (2015). Turning mirror-image oligonucleotides into drugs: the evolution of Spiegelmer ${ }^{\circledR}$ therapeutics. Drug Discov Today 20:147-155.

17. Ishiguro A, T Akiyama, $\mathrm{H}$ Adachi, J Inoue and Y Nakamura. (2011). Therapeutic potential of anti-interleukin-17A aptamer: suppression of interleukin-17A signaling and attenuation of autoimmunity in two mouse models. Arthritis Rheum 63:455-466.

18. Healy JM, SD Lewis, M Kurz, RM Boomer, KM Thompson, C Wilson and TG McCauley. (2004). Pharmacokinetics and biodistribution of novel aptamer compositions. Pharm Res 21:2234-2246.

19. Maione F, N Pashalidis, N Mascolo, N Dufton, M Perretti and F D' Acquisto (2009). Interleukin 17 sustains rather than induces inflammation. Biochem Pharmacol 77:878-887.

20. Kang H, JK Park, YB Seu and SK Hahn. (2007). A novel branch-type PEGylation of aptamer therapeutics. Key Eng Mater 342-343:529-532.

21. Yamaoka T, Y Tabata and Y Ikeda. (1994). Distribution and tissue uptake of poly(ethylene glycol) with different molecular weights after intravenous administration to mice. J Pharm Sci 83:601-606.

22. Lee JF, GM Stovall and AD Ellington. (2006). Aptamer therapeutics advance. Curr Opin Chem Biol 10:282-289.

23. Palframan R, M Airey, A Moore, A Vugler and A Nesbitt. (2009). Use of biofluorescence imaging to compare the distribution of certolizumab pegol, adalimumab, and infliximab 
in the inflamed paws of mice with collagen-induced arthritis. J Immunol Methods 348:36-41.

24. Mcginness K, JL Diener, RG Schaub and K Thompson. (2010). Patent US2010/023599; PCT/WO/10/091396.

25. Siller-Matula JM, Y Merhi, JF Tanguay, D Duerschmied, DD Wagner, KE McGinness, PS Pendergrast, JK Chung, X Tian, RG Schaub and B Jilma. (2012). ARC15105 is a potent antagonist of von Willebrand Factor mediated platelet activation and adhesion. Arterioscler Thromb Vasc Biol 32:902-909.

26. Miossec P and JK Kolls. (2012). Targeting IL-17 and TH17 cells in chronic inflammation. Nat Rev Drug Discov 11: 763-776.

27. Chen L, DQ Li, J Zhong, XL Wu, Q Chen, H Peng and SQ Liu. (2011). IL-17RA aptamer-mediated repression of IL-6 inhibits synovium inflammation in a murine model of osteoarthritis. Osteoarthritis Cartilage 19:711-718.

28. Otaki N, A Sasaki, S Hiramoto, M Nagamine, S Mori, T Kayo, K Hota, M Takahashi, K Haruta and Y Nakamura. (2014) Improvement of the stability of RNA aptamers against Interleukin-17A. Arthritis Rheum 66 (Suppl. 10): S332.

29. Uyttenhove C and J Van Snick. (2006). Development of an anti-IL-17A auto-vaccine that prevents experimental autoimmune encephalomyelitis. Eur J Immunol 36:2868-2874.

30. Iwanami K, I Matsumoto, Y Tanaka-Watanabe, A Inoue, M Mihara, Y Ohsugi, M Mamura, D Goto, S Ito, et al. (2008). Crucial role of the interleukin-6/interleukin-17 cytokine axis in the induction of arthritis by glucose-6-phosphate isomerase. Arthritis Rheum 58:754-763.

31. Rizzo HL, S Kagami, KG Phillips, SE Kurtz, SL Jacques and A Blauvelt. (2011). IL-23-mediated psoriasis-like epidermal hyperplasia is dependent on IL-17A. J Immunol 186: 1495-1502.

32. Langley RG, BE Elewski, M Lebwohl, K Reich, CEM Griffiths, K Papp, L Puig, H Nakagawa, L Spelman, et al.
(2014). Secukinumab in plaque psoriasis-results of two phase 3 trials. N Engl J Med 371:326-338.

33. Griffiths CEM, K Reich, M Lebwohl, $P$ van de Kerkhof, $C$ Paul, A Menter, GS Cameron, J Erickson, L Zhang, et al. (2015). Comparison of ixekizumab with etanercept or placebo in moderate-to-severe psoriasis (UNCOVER-2 and UNCOVER-3): results from two phase 3 randomised trials. Lancet 386:541-551.

34. Baeten D, J Sieper, J Braun, X Baraliakos, M Dougados, P Emery, A Deodhar, B Porter, R Martin, et al. (2015). Secukinumab, an interleukin-17A inhibitor, in ankylosing spondylitis. N Engl J Med 373:2534-2548.

35. Osborn BL, HS Olsen, B Nardelli, JH Murray, JXH Zhou, A Garcia, G Moody, LS Zaritskaya and C Sung. (2002). Pharmacokinetic and pharmacodynamic studies of a human serum albumin-interferon- $\alpha$ fusion protein in cynomolgus monkeys. J Pharmacol Exp Ther 303:540-548.

36. Takeuchi T, N Miyasaka, S Kawai, N Sugiyama, H Yuasa, N Yamashita, N Sugiyama, LC Wagerle, B Vlahos and J Wajdula. (2015). Pharmacokinetics, efficacy and safety profiles of etanercept monotherapy in Japanese patients with rheumatoid arthritis: review of seven clinical trials. Mod Rheumatol 25:173-186.

Address correspondence to: Kazuhiko Haruta, PhD $R \& D$ Center Zenyaku Kogyo Co., Ltd. 2-33-7 Oizumi, Nerima Tokyo 178-0062 Japan

E-mail: kazuhiko_haruta@mail.zenyaku.co.jp

Received for publication May 8, 2016; accepted after revision June 21, 2016. 